\title{
Modelling cometary meteoroid stream traverses of the Martian Moons eXploration (MMX) spacecraft en route to Phobos
}

\author{
Harald Krüger ${ }^{1,2^{*}}$ (D), Masanori Kobayashi ${ }^{2}$, Peter Strub ${ }^{1,3}$, Georg-Moragas Klostermeyer ${ }^{3}$, Maximilian Sommer ${ }^{3}$,
} Hiroshi Kimura², Eberhard Grün ${ }^{4,5}$ and Ralf Srama ${ }^{3,6}$

\begin{abstract}
The Martian Moons Exploration (MMX) spacecraft is a JAXA mission to Mars and its moons Phobos and Deimos. MMX will be equipped with the Circum-Martian Dust Monitor (CMDM) which is a newly developed light-weight (650 g) large area $\left(1 \mathrm{~m}^{2}\right)$ dust impact detector. Cometary meteoroid streams (also referred to as trails) exist along the orbits of comets, forming fine structures of the interplanetary dust cloud. The streams consist predominantly of the largest cometary particles (with sizes of approximately $100 \mu \mathrm{m}$ to $1 \mathrm{~cm}$ ) which are ejected at low speeds and remain very close to the comet orbit for several revolutions around the Sun. The Interplanetary Meteoroid Environment for eXploration (IMEX) dust streams in space model is a new and recently published universal model for cometary meteoroid streams in the inner Solar System. We use IMEX to study the detection conditions of cometary dust stream particles with CMDM during the MMX mission in the time period 2024 to 2028. The model predicts traverses of 12 cometary meteoroid streams with fluxes of $100 \mu \mathrm{m}$ and bigger particles of at least $10^{-3} \mathrm{~m}^{-2}$ day $\mathrm{y}^{-1}$ during a total time period of approximately 90 days. The highest flux of $0.15 \mathrm{~m}^{-2}$ day ${ }^{-1}$ is predicted for comet 114P/Wiseman-Skiff in October 2026. With its large detection area and high sensitivity CMDM will be able to detect cometary meteoroid streams en route to Phobos. Our simulation results for the Mars orbital phase of MMX also predict the occurrence of meteor showers in the Martian atmosphere which may be observable from the Martian surface with cameras on board landers or rovers. Finally, the IMEX model can be used to study the impact hazards imposed by meteoroid impacts onto large-area spacecraft structures that will be particularly necessary for crewed deep space missions.
\end{abstract}

Keywords: Comets, Meteoroid trails, Meteoroid streams, Interplanetary dust, Martian moons, Phobos, Deimos, Martian Moons Exploration, MMX

\section{Introduction}

The Japanese Space Exploration Agency (JAXA) is planning to send the Martian Moons Exploration (MMX) spacecraft to the Martian moons Phobos and Deimos and to bring samples from Phobos to Earth (Kuramoto et al. 2018). The launch of MMX is planned for 2024, and Phobos sample arrival at Earth is scheduled for 2029.

*Correspondence: krueger@mps.mpg.de

2 PERC, Chiba Institute of Technology, Narashino, Japan

Full list of author information is available at the end of the article
MMX will be equipped with the large area CircumMartian Dust Monitor (CMDM) which has a sensitive area of $1 \mathrm{~m}^{2}$ (Kobayashi et al. 2018a). The primary goals of the CMDM dust investigations are to understand dust processes in the circum-Martian environment, including the search for a proposed but still undiscovered circumMartian dust ring (Soter 1971; Hamilton 1996; Krivov and Hamilton 1997; Ishimoto et al. 1997; Showalter et al. 2006). A second goal is the measurement of interplanetary and interstellar dust (Kobayashi et al. 2018a) as well as cometary meteoroid streams. 
The prominent dust tail of a comet predominantly consists of small submicrometer-sized particles that are blown out by the solar radiation pressure force. Larger dust particles with sizes of approximately $100 \mu \mathrm{m}$ to $1 \mathrm{~cm}$ are ejected from the cometary nucleus at low speeds and remain very close to the comet orbit for several revolutions around the Sun (Agarwal et al. 2010). They slowly spread in the comet orbit as a result of small differences in orbital period, and they form a tubular structure along the orbit of the parent comet filled with dust, called a dust stream or trail. Particles in the intermediate size range from approximately $1 \mu \mathrm{m}$ to $100 \mu \mathrm{m}$ are still subject to radiation pressure, thus migrating away from the comet's orbital position, but they stay on trajectories similar to the orbit of their parent comet for some time.

Dust trails in the vicinity of comets were first observed by the Infrared Astronomical Satellite (IRAS; Sykes et al. 1986; Sykes and Walker 1992). In subsequent infrared observations, at least $80 \%$ of the observed Jupiter-family comets were associated with dust trails which can thus be considered one of their generic features (Reach et al. 2007). A recent review of the present knowledge on cometary dust, including dust trails, was given by Levasseur-Regourd et al. (2018).

These meteoroid trails form fine structures superimposed on the interplanetary background dust cloud. When the Earth intercepts a cometary trail, the particles collide with the atmosphere and show up as meteors and fireballs with hourly rates significantly enhanced over the sporadic meteor background flux (Koschny et al. 2019, and references therein). Effects of meteoroid impacts were also observed on the Earth's Moon and on other planets (see Christou et al. 2019, for a comprehensive review). Meteor trails can impose safety risks for Earth orbiting and interplanetary spacecraft. Although the impact probability per spacecraft is small, spacecraft anomalies very likely related to meteoroid impacts were observed in the past (Caswell et al. 1995; Cameron et al. 2004). The risk is considered sufficiently high to occasionally affect crewed spaceflight operations (Beech et al. 1995).

In the past, the detection of meteoroid trails by spacecraft-based in situ dust detectors was hindered by the relatively small sensitive area of the instruments and low particle fluxes in the trails. Only recently could a small number of impacts of likely cometary meteoroid particles be identified in the data set collected by the in situ dust instruments onboard the Helios spacecraft in the inner Solar System in the 1970s (Altobelli et al. 2006; Krüger et al. 2020). Interestingly, the identification of trail particles was only possible because Helios traversed the same region of space and thus, the same cometary trails, many times which significantly increased the chances to detect trail particles with these relatively small impact ionization dust sensors. MMX with its large area CMDM dust detector will be excellently equipped to make the first undisputable in situ detections of cometary meteoroid trails.

Cometary dust trails can be simulated with the Interplanetary Meteoroid Environment for eXploration (IMEX) dust streams in space model which was developed under contract by the European Space Agency (Soja et al. 2015b, a, 2019). IMEX is a new universal and physical model for dust dynamics and orbital evolution that simulates cometary dust trails in the inner Solar System. The model follows the trails of 420 comets and is ideal for studying meteor streams and cometary dust trails as measured by in situ detectors and observed in infrared images. A recent review of presently existing up to date models for dust in interplanetary space in the inner solar system can be found in Grün et al. (2019).

We use the IMEX model to study future cometary trail traverses by the MMX spacecraft during its interplanetary transfer to Mars and during its Martian orbital phase. After a brief description of the MMX mission and the CMDM instrument, we give an overview of the IMEX model. Next, we present and discuss the results of our IMEX simulations and, finally, we summarise our conclusions.

\section{MMX mission and Circum-Martian Dust Monitor}

Building on the experience of the asteroid sample return spacecraft Hayabusa and Hayabusa 2, JAXA is planning to launch the Martian Moons eXploration (MMX) spacecraft to Phobos and Deimos (Kuramoto et al. 2018, 2019). The mission will perform the first landing on one of the Martian moons, Phobos, collect samples from its surface, and return them to Earth. In addition to the returned samples, the mission will perform close-up remote sensing and in situ measurements at the two moons, and deploy a small rover on the Phobos surface. The launch of MMX is planned for 2024, the spacecraft will be in Mars orbit from 2025 to 2028, and the return module will arrive at Earth in 2029.

For its transfer to Mars MMX will use a low-energy transfer orbit, which takes about one year to reach the planet. This trajectory takes more time than a classical Hohmann transfer but significantly reduces the amount of fuel needed (Topputo and Belbruno 2015; Ogawa et al. 2019). After Mars orbit insertion, MMX will initially orbit the planet on a trajectory near Phobos' orbit. Subsequently the spacecraft will be injected into a quasisatellite orbit around the moon with a minimum altitude above the Phobos surface of $20 \mathrm{~km}$ or less (Kuramoto et al. 2019). MMX will be a 3-axis stabilised spacecraft throughout the entire mission. 
One of the scientific instruments on board MMX will be the Circum-Martian Dust Monitor (CMDM; Kobayashi et al. 2018a). It is a large area dust detector with $1 \mathrm{~m}^{2}$ sensor area. For dust detection CMDM will use the outermost polyimide film of the spacecraft multilayer insulation (MLI), which is a thermal blanket of the spacecraft. The sensor will use the MLI on one flat side of the spacecraft and have a field-of-view of $2 \pi$. Additional piezoelectric elements will be mounted on the MLI surface. A dust particle hitting or penetrating the MLI surface generates a stress wave that subsequently propagates through the MLI film. It can be detected by one or more of the piezoelectric sensors, and the position of the impact on the MLI can be derived from the differences between the arrival times of the signals measured on the multiple pick-up sensors. From the derived position of the crater or penetration location, it will be possible to distinguish whether it is a dust impact or noise event (Kobayashi et al. 2016). Information about the projectile's mass and impact speed can be derived from the waveform of the signal. At $10 \mathrm{~km} \mathrm{~s}^{-1}$ impact speed the instrument can detect impacts of particles as small as approximately $3 \mu \mathrm{m}$ in radius.

\section{IMEX cometary trails model}

In order to identify time intervals when the MMX spacecraft will traverse cometary meteoroid trails, we use the IMEX dust streams in space model (Soja et al. 2015b, a, 2019). The model generates trails for 362 Jupiter-family, 40 Halley-type, and 18 Encke-type comets available in the JPL Small Body Database (SBDB) as of 1 August 2013, which have perihelion distances $q<3 \mathrm{AU}$, semimajor axes $a<30 \mathrm{AU}$, and defined total visual magnitudes.

Particles are emitted when the comet is in the inner Solar System, taking into account perihelion passages between the years 1700 and 2080 for Encke-type comets, and between 1850 and 2080 for Jupiter-family and Halley-type comets, respectively. This reflects the fact that the most recently released dust is expected to be most important, and also the maximum size of the database that could be maintained at the time when the model was developed.

For each passage through the inner Solar System within $3 \mathrm{AU}$ of the Sun of each comet (which we refer to as apparition in the following), particles are emitted from the sunlit hemisphere of the comet nucleus within the time ranges specified above. About 28,000 particles are ejected per comet per apparition for Halley-type comets; and about 14,000 for other comets.

The dust ejection is described by the comet emission model of Crifo and Rodionov (1997). The model assumes the dust emission to be driven by water gas production within $3 \mathrm{AU}$ of the Sun. It estimates the water production rate using the visual magnitude, and a gas-to-dust ratio (Jorda et al. 2008). The JPL Small Body Database provides total and nuclear magnitudes.

Dust-to-gas mass ratios can be estimated for individual comets, and they mostly range from 0.1 to 3 , though higher values are possible. They appear to be dependent on heliocentric distance (A'Hearn et al. 1995). Given the large uncertainties in dust-to-gas ratios, the model uses a value of 1 . Deviations from this can be considered in the analysis of individual comets.

The IMEX model uses the mass distribution model of Divine et al. (1987) and Agarwal et al. (2007, 2010), with model parameters given by Soja et al. (2015b). The mass distribution covers the range from $10^{-8} \mathrm{~kg}$ to $10^{-2} \mathrm{~kg}$, separated into eight mass bins (approximately corresponding to $100 \mu \mathrm{m}$ to $1 \mathrm{~cm}$ particle radius; Soja et al. 2015b). The particle density is assumed to be $\rho=1000 \mathrm{~kg} \mathrm{~m}^{-3}$. For comets with unknown radius a value of $1 \mathrm{~km}$ is assumed (Soja et al. 2015b).

The trajectory of each emitted particle is integrated individually including solar gravity, planetary perturbations, solar radiation pressure and Poynting-Robertson drag. The model calculates the impact velocity for each individual particle onto the spacecraft as well as dust number density and flux. A detailed model description including an application to the trail of comet 67P/Churyumov-Gerasimenko was given by Soja et al. (2015b). We use the IMEX model to identify time intervals when MMX will traverse the meteoroid trails of comets between September 2024 and August 2028 which covers the presently planned interplanetary transfer to Mars and the Martian orbital phase of the spacecraft.

For the interplanetary transfer from Earth to Mars we use a preliminary MMX trajectory provided by ISAS/ JAXA. It is the nominal trajectory in the mission analysis performed by Campagnola et al. (2018) with a launch in 2024. For the Martian orbital phase of MMX we use Mars as the target for our simulations. We do not consider the interplanetary return to Earth in this work. CMDM will be mounted on the spacecraft module that will return to Earth and be able to collect data during this mission phase, however, we do not have a trajectory for this mission phase available at the present time. Given that IMEX is a time-dependent model, the use of the actual spacecraft trajectory is a prerequisite to make reliable predictions for the occurrence of trail traverses and the dust fluxes during such traverses. 


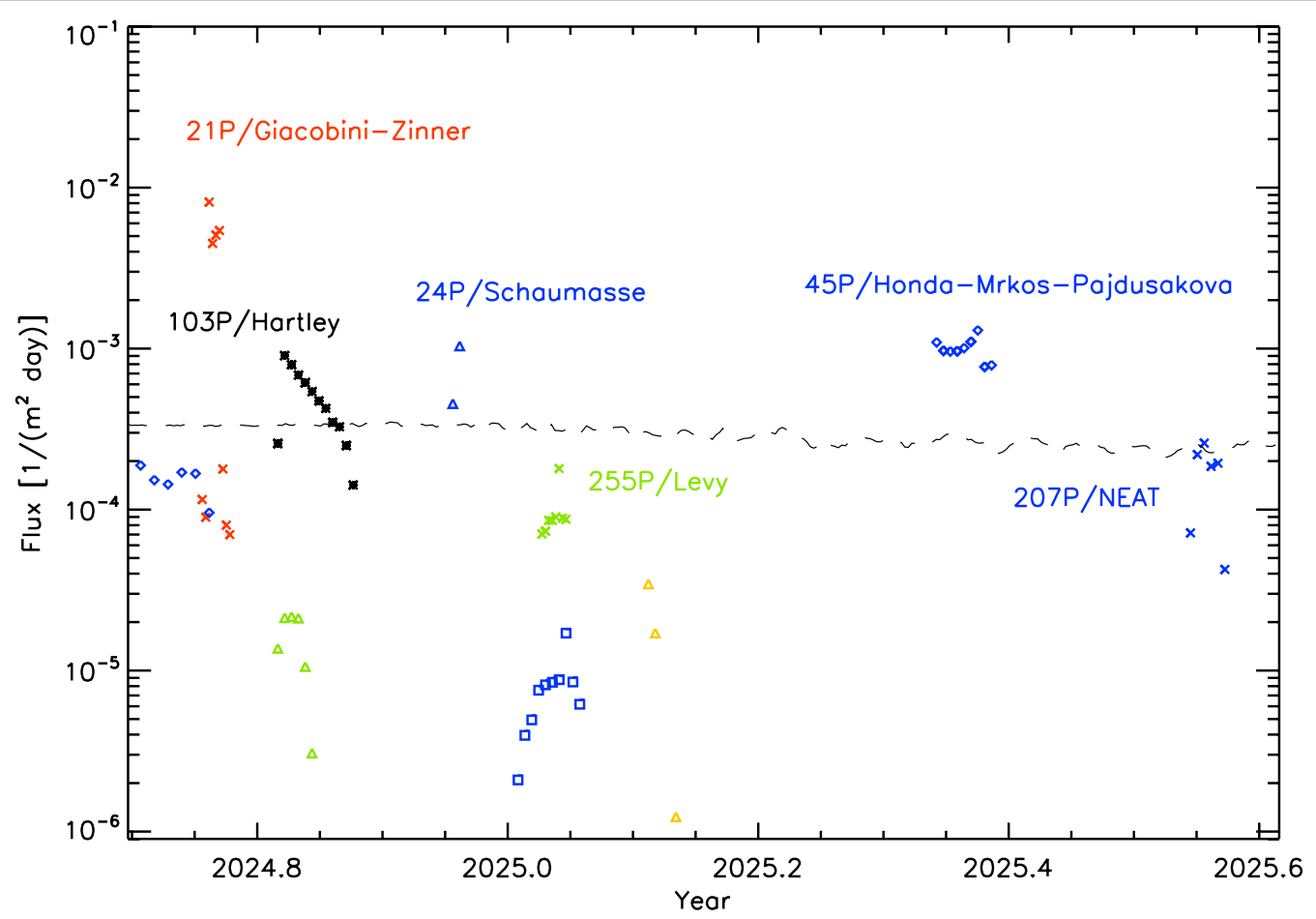

Fig. 1 Simulated dust fluxes for cometary meteoroid trails intercepted by the MMX spacecraft during its interplanetary transfer to Mars (cf. Fig. 2). Simulated particles are larger than $100 \mu \mathrm{m}$. Symbols and colours distinguish individual comets: red crosses: 21P/Giacobini-Zinner; blue triangles: 24P/Schaumasse; blue diamonds: 45P/H-M-P; black asterisks: 103P/Hartley; green crosses: 255P/Levy; blue crosses: 207P/NEAT; green triangles: 249P/LINEAR; blue squares: 263P/Gibbs; yellow triangles: 41P/Tuttle-Giacobini-Kresák. The simulations were performed with a 1-day step size for 21P, and with a 2-day step size for all other comets. The dashed line shows the simulated flux of sporadic interplanetary dust particles, see text for details

\section{Results}

Figure 1 shows the simulated dust fluxes during cometary trail traverses of MMX along its interplanetary transfer to Mars. The MMX trajectory for this mission phase is shown in Fig. 2, and details of these trail traverses are given in Table 1.

The highest flux during the transfer to Mars is predicted for comet 21P/Giacobini-Zinner: For a trail traverse in October 2024 the model predicts a maximum flux of $8 \cdot 10^{-3} \mathrm{~m}^{-2} \mathrm{day}^{-1}$. It is the highest meteoroid trail flux expected during the interplanetary transfer phase of MMX. For comets 103P/Hartley, 24P/Schaumasse and 45P/Honda-Mrkos-Pajdušáková (hereafter 45P/H-M-P) the predicted fluxes are approximately $10^{-3} \mathrm{~m}^{-2}$ day ${ }^{-1}$ in late October 2024, mid December 2024 and in May 2025. For these four comets the time intervals with fluxes of approximately $10^{-3} \mathrm{~m}^{-2} \mathrm{day}^{-1}$ or higher last between two (24P/Schaumasse and 103P/Hartley) and 12 days $(45 \mathrm{P} / \mathrm{H}-\mathrm{M}-\mathrm{P})$, adding up to a total of 20 days. The model finds 23 additional comets with fluxes below $10^{-3} \mathrm{~m}^{-2}$ day $^{-1}$ which we do not consider relevant. MMX will cross the trail of 45P/H-M-P twice, namely in September 2024 and in May 2025.
Figure 2 shows the locations of trail traverses and the impact directions of trail particles onto the spacecraft in the spacecraft-centered reference frame projected onto the ecliptic plane. The particle impact speeds for the above four comets are in the range $10 \mathrm{~km} \mathrm{~s}^{-1} \lesssim\left|\mathrm{v}_{\text {imp }}\right| \lesssim 20 \mathrm{~km} \mathrm{~s}^{-1}$. Table 1 gives the impact speeds together with the impact directions of the particles. The latter are given as the upstream direction in the spacecraft frame of reference of the particle flow which corresponds to the radiant of a meteor stream in a planetary atmosphere.

For the MMX Mars Orbital Phase the simulated dust fluxes are shown in Fig. 3. For seven comets with a total of nine trail traverses the predicted fluxes exceed $10^{-3} \mathrm{~m}^{-2} \mathrm{day}^{-1}$, see also Table 1 . The time intervals with fluxes above this limit last between 2 and 15 days, adding up to a total of 70 days. The comet trail traverse with the highest predicted flux is the one of 114P/WisemanSkiff with a maximum of $1.5 \cdot 10^{-1} \mathrm{~m}^{-2}$ day $^{-1}$. However, this trail is rather collimated, leading to a short traverse which lasts only three days. The model finds 72 additional comets with fluxes below $10^{-3} \mathrm{~m}^{-2} \mathrm{day}^{-1}$. As for 


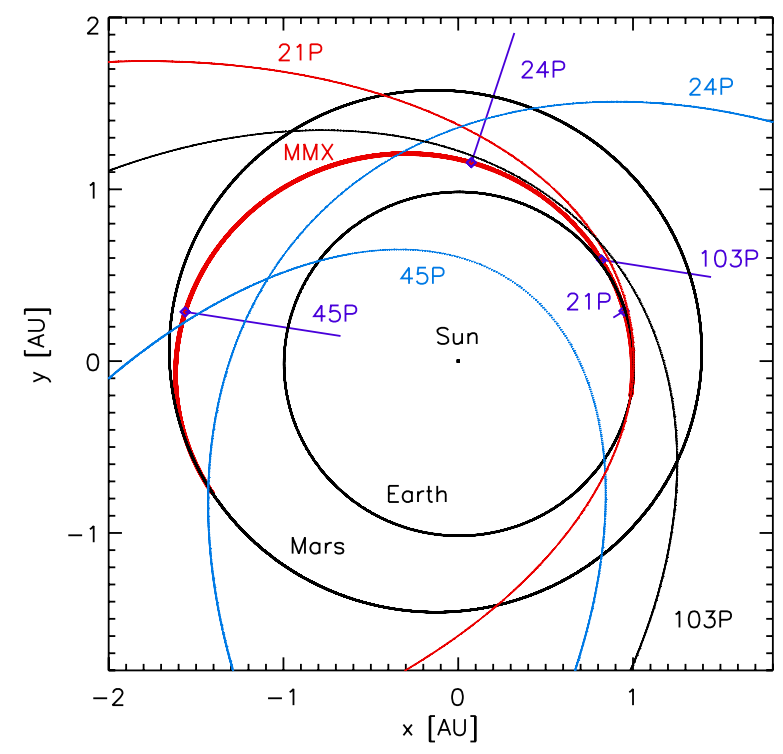

Fig. 2 Interplanetary trajectory of MMX (thick red line). Blue diamonds indicate the crossings of cometary trails with predicted dust fluxes of at least $10^{-3} \mathrm{~m}^{-2} \mathrm{day}^{-1}$ (cf. Fig. 1). Blue lines indicate the approach direction (speed vector) of trail particles onto the spacecraft in the spacecraft-centered reference frame projected onto the X-Y plane (cf. Table 1). The X-Y plane is the ecliptic plane with vernal equinox oriented towards the $+X$ direction. The comet orbits are superimposed as thin lines

the interplanetary transfer phase, we do not consider them relevant.

Figures 1 and 3 also show the flux of sporadic interplanetary particles predicted by the Interplanetary Meteoroid Engineering Model (IMEM) developed by Dikarev et al. (2005). For the IMEM simulations we assumed a spinning flat plate sensor. Given that the actual CMDM orientation on board the 3-axis-stabilized MMX spacecraft during the mission is not known at the present time, this is a reasonable approximation. We adopted the same particle size range as for the IMEX meteoroid trail simulations $\left(10^{-8} \mathrm{~kg}\right.$ to $\left.10^{-2} \mathrm{~kg}\right)$ so that the fluxes can be directly compared with the fluxes predicted for the meteoroid trail traverses (Due to artefacts in the predicted fluxes for a lower mass cutoff at $10^{-8} \mathrm{~kg}$ caused by the parametrisation of the cometary particle populations in the IMEM model, we used a slightly lower boundary of $4 \cdot 10^{-9} \mathrm{~kg}$ for the simulations and scaled the curves shown in Figs. 1 and 3 by a factor of 2.5 that we derived from the particle size distribution). The highest predicted trail fluxes typically exceed the flux of sporadic interplanetary particles of the same size by approximately one (during interplanetary transfer) to two orders of magnitude (during Mars orbital phase). We ignore interstellar dust because it does not make any significant contribution to the dust fluxes in this size range (Strub et al. 2019). Fluxes expected for smaller interplanetary and interstellar particles down to the detection limit of CMDM as predicted by IMEM are given by Kobayashi et al. (2018a).

As for the interplanetary transfer, the locations of trail traverses and impact directions of trail particles are shown in Fig. 4. The particle impact speeds are in the range $10 \mathrm{~km} \mathrm{~s}^{-1} \lesssim\left|\mathrm{v}_{\text {imp }}\right| \lesssim 22 \mathrm{~km} \mathrm{~s}^{-1}$. Again the impact directions and speeds are given in Table 1. Strictly speaking, impact speeds and directions refer to an observer on Mars because we have used Mars as the target for our simulations. The orbital elements of the identified comets are given in Table 2 in the Appendix. All comets identified in our analysis are Jupiter Family Comets with orbital periods between 5.2 and 13.8 years.

Column 6 in Table 1 lists the total number of expected particle detections with CMDM during the traverse of each comet's trail (fluence). All these numbers are below 1 , with the highest values being the fluences of approximately 0.3 for comets 19P/Borrelly and 114P/Wiseman-Skiff. It has to be noted that these fluences refer to particle sizes of approximately $100 \mu \mathrm{m}$ and bigger, while CMDM can detect particles at least one order of magnitude smaller. These smaller particles are not covered by the IMEX model and, thus, the fluences detectable with CMDM are expected to be significantly higher. We will discuss this in the next Section.

\section{Discussion}

Individual trail particles originating from comets $45 \mathrm{P} / \mathrm{H}$ M-P and 72P/Denning-Fujikawa were likely detected by the Helios dust instruments already in the 1970s (Altobelli et al. 2006; Krüger et al. 2020). IMEX predicts a flux of approximately $10^{-2} \mathrm{~m}^{-2}$ day ${ }^{-1}$ for the traverses of these meteoroid trails by Helios. It has to be emphasised that the dust sensors on board Helios had a combined effective sensor area for trail particle detections of only about $30 \mathrm{~cm}^{2}$. However, Helios traversed the same region of space, and thus the same comet trail, ten times, which increased the detection probability also by a factor of ten. Considering that the sensor area of CMDM will be larger by a factor of more than 300 while the dust fluxes predicted by IMEX are similar within an order of magnitude, we conclude that CMDM has a very good chance to detect cometary trail particles as well.

Our analysis of the Helios dust data showed an offset by a few days for some dust particle detections as compared to the times predicted by the IMEX model (Krüger et al. 2020). Various reasons may be responsible for such an offset which include shortcomings of the IMEX model like, for example, the dust ejection model employed which may not fully cover all relevant aspects of dust ejection, or the particle trajectory computation 


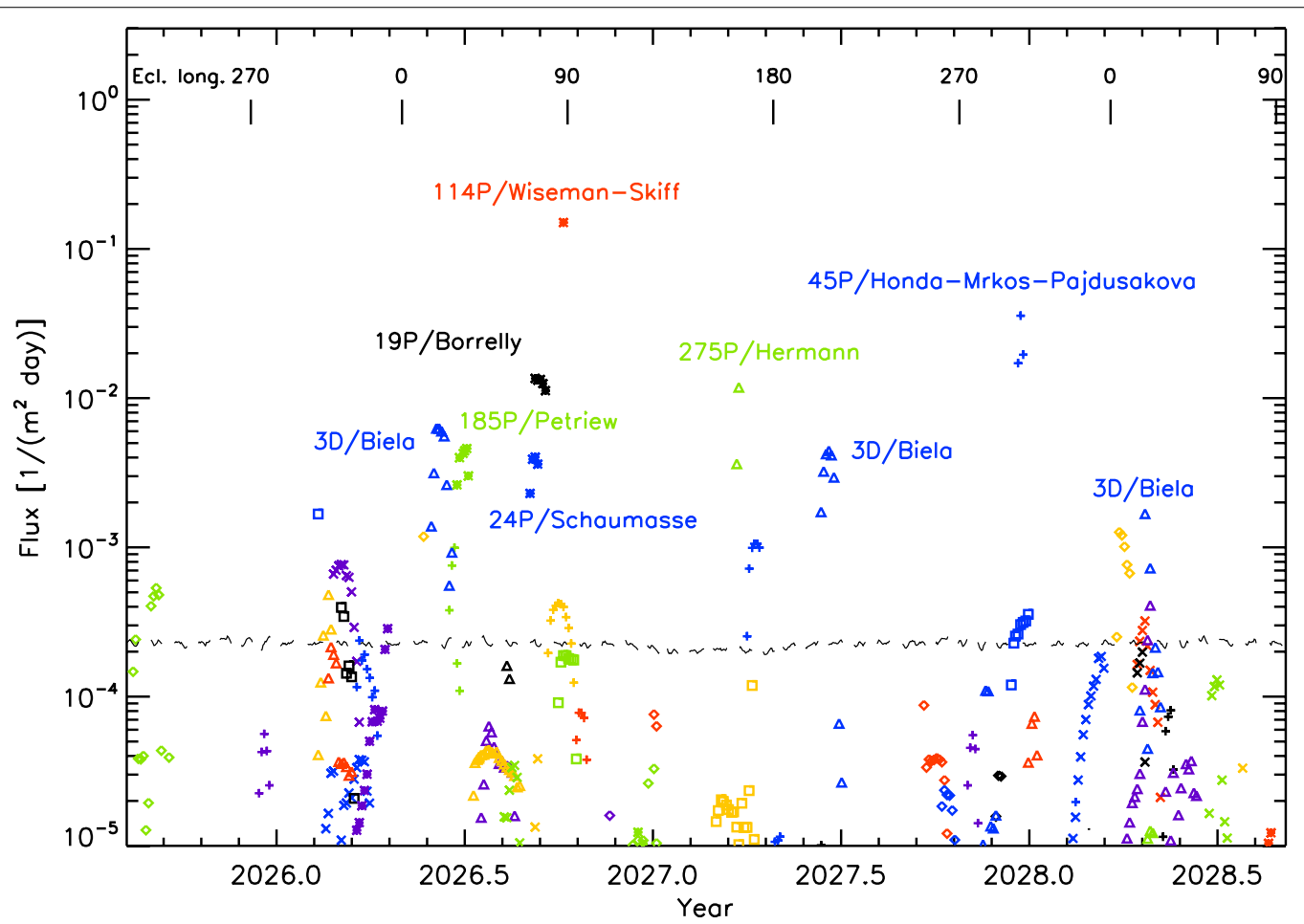

Fig. 3 Simulated dust fluxes for cometary meteoroid trails intercepted by the MMX spacecraft during its orbital phase at Mars (cf. Fig. 4). Simulated particles are larger than $100 \mu \mathrm{m}$. Symbols and colors distinguish individual comets: red asterisks: 114P/Wiseman-Skiff; black asterisks: 19P/Borrelly; blue triangles: 3D/Biela; blue crosses: 45P/H-M-P; green triangles: 275P/Hermann; green asterisks: 185P/Petriew; blue asterisks: 24P/Schaumasse; blue squares: 10P/Tempel 2. The remaining symbols refer to other comets forming a very low background flux. The simulations were performed with a 2.5-day step size. The ecliptic longitude of Mars is indicated at the top, and the dashed line shows the simulated flux of interplanetary dust particles, see text for details

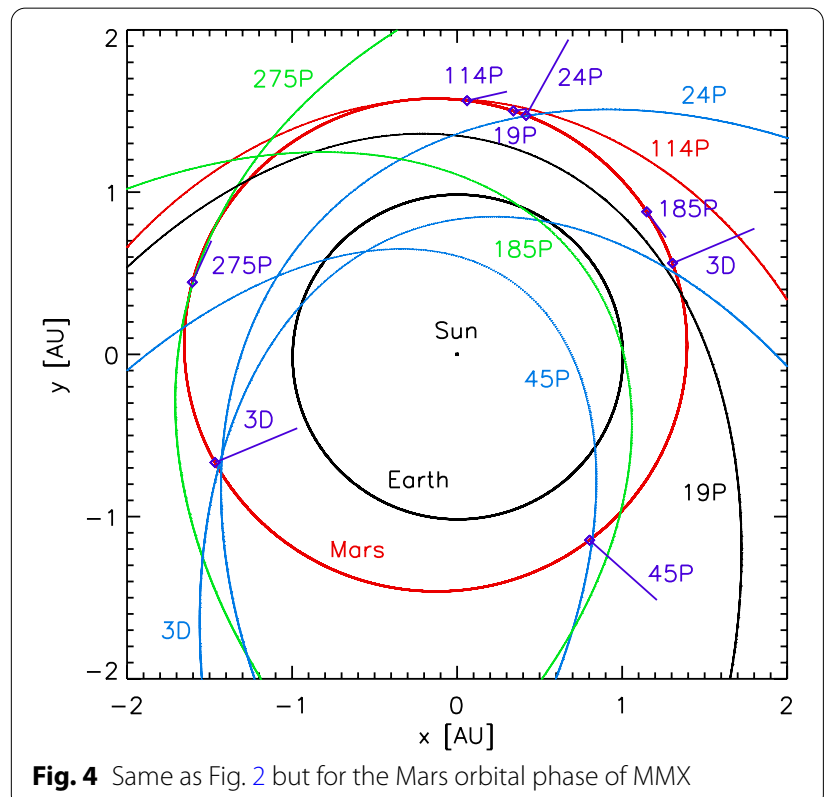

which follows the particle motion only for up to 300 years. Furthermore, the model simulates particles with masses $10^{-8} \mathrm{~kg}$ and bigger, corresponding to particle radii above approximately $100 \mu \mathrm{m}$ while the masses of the particles detected by Helios as derived from the calibration of the Helios dust instruments were at least four orders of magnitude less massive, corresponding to particle radii of a few micrometers to about $10 \mu \mathrm{m}$ (Note that the Helios dust instruments had a detection threshold for dust particles with masses of approximately $3 \cdot 10^{-16} \mathrm{~kg}$ at an impact speed of $10 \mathrm{~km} \mathrm{~s}^{-1}$ (Grün et al. 1980), corresponding to a particle radius of approximately $0.3 \mu \mathrm{m}$ ). Such smaller particles are more susceptible to radiation pressure and Poynting-Robertson drag than the larger ones which leads to an increased particle dispersion in space. From our analysis of the Helios data we concluded that the size (mass) of the impactors as derived from the instrument calibration may be significantly underestimated, even more so if cometary particles are fluffy aggregates as shown, for example, by the results from the Rosetta mission to comet 67P/Churymov-Gerasimenko (Güttler et al. 2019; Kimura et al. 2020b, a). A more detailed discussion is given by Krüger et al. (2020). To 
Table 1 Results from the IMEX cometary dust trails simulations for MMX

\begin{tabular}{|c|c|c|c|c|c|c|c|c|c|c|c|}
\hline \multirow[t]{3}{*}{ Comet } & \multirow{3}{*}{$\begin{array}{l}\text { Time interval with flux } \\
\geq 10^{-3} \mathrm{~m}^{-2} \text { day }^{-1}\end{array}$} & \multirow{3}{*}{$\begin{array}{l}\text { Duration } \\
\text { [days] }\end{array}$} & \multirow{3}{*}{$\begin{array}{l}\text { Day with } \\
\text { maximum flux }\end{array}$} & \multirow{3}{*}{$\begin{array}{l}\text { Maximum } \\
\text { flux } \\
{\left[\mathrm{m}^{-2} \text { day }^{-1}\right]}\end{array}$} & \multirow[t]{2}{*}{ Fluence } & \multicolumn{4}{|c|}{ Impact speed at maximum flux } & \multicolumn{2}{|c|}{$\begin{array}{l}\text { Impact } \\
\text { direction }\end{array}$} \\
\hline & & & & & & $\left|v_{\text {imp }}\right|$ & $v_{x}$ & $v_{y}$ & $v_{z}$ & $\lambda_{\text {ecl }}$ & $\beta_{\mathrm{ecl}}$ \\
\hline & & & & & {$\left[m^{-2}\right]$} & {$\left[\mathrm{km} \mathrm{s}^{-1}\right]$} & {$\left[\mathrm{kms}^{-}\right]$} & {$\left[\mathrm{kms}^{-}\right]$} & {$\left[\mathrm{km} \mathrm{s}^{-}\right]$} & {$\left[{ }^{\circ}\right]$} & {$\left[^{\circ}\right]$} \\
\hline (1) & $(2)$ & (3) & (4) & (5) & $(6)$ & (7) & (8) & (9) & $(10)$ & $(11)$ & $(12)$ \\
\hline \multicolumn{12}{|c|}{ Interplanetary Transfer Phase } \\
\hline 21P/Giacobini-Zinner & 04.10.2024-11.10.2024 & 8 & 05.10 .2024 & 0.0082 & 0.013 & 20.2 & 1.4 & 0.9 & -20.1 & 212 & 85 \\
\hline 24P/Schaumasse & 14.12.2024-16.12.2024 & 2 & 16.12 .2024 & 0.0011 & 0.002 & 20.4 & -5.6 & -17.1 & 9.6 & 72 & -28 \\
\hline 45P/H-M-P & 05.05.2025-17.05.2025 & 13 & 17.05 .2025 & 0.0013 & 0.015 & 17.6 & -17.4 & 2.7 & 0.0 & 351 & 0 \\
\hline 103P/Hartley & $25.10 .2024-26.10 .2024$ & 2 & 26.10 .2024 & 0.0009 & 0.001 & 10.0 & -7.0 & 1.1 & -7.1 & 351 & 45 \\
\hline \multicolumn{12}{|l|}{ Mars Orbital Phase } \\
\hline 3D/Biela & 30.05.2026-15.06.2026 & 17 & 10.06 .2026 & 0.0064 & 0.006 & 20.5 & -18.5 & -8.0 & -3.8 & 23 & 11 \\
\hline 3D/Biela & 13.06.2027-25.06.2027 & 13 & 20.06 .2027 & 0.0045 & 0.050 & 20.4 & -18.5 & -7.8 & 3.6 & 23 & -10 \\
\hline 19P/Borrelly & 08.09.2026-18.09.2026 & 11 & 15.09 .2026 & 0.0310 & 0.332 & 15.7 & -2.4 & -0.3 & 15.5 & 8 & -81 \\
\hline 24P/Schaumasse & 02.09.2026-13.09.2026 & 12 & 09.09 .2026 & 0.0039 & 0.041 & 16.4 & -7.6 & -13.9 & 4.1 & 61 & -14 \\
\hline 45P/H-M-P & 21.12.2027-26.12.2027 & 6 & 24.12 .2027 & 0.0355 & 0.120 & 22.3 & -16.6 & 15.0 & -0.2 & 318 & 1 \\
\hline 114P/Wiseman-Skiff & $04.10 .2026-07.10 .2026$ & 3 & 06.10 .2026 & 0.1535 & 0.300 & 11.0 & -4.9 & -1.1 & -9.9 & 13 & 63 \\
\hline 185P/Petriew & 24.06.2026-05.07.2026 & 12 & 04.07 .2026 & 0.0046 & 0.037 & 10.7 & -2.3 & 3.1 & -10.0 & 306 & 69 \\
\hline 275P/Hermann & $22.03 .2027-25.03 .2027$ & 4 & 24.03 .2027 & 0.0110 & 0.034 & 12.3 & -2.6 & -5.7 & -10.6 & 66 & 59 \\
\hline
\end{tabular}

The time interval of the trail traverse together with the predicted maximum flux (of $100 \mu \mathrm{m}$ and bigger particles) are given in columns (2) to (5). Column (6) gives the fluence on to a $1 \mathrm{~m}^{2}$ detector detectable during the time interval given in column (2). Columns (7) to (10) give the speed vector, while columns (11) and (12) give the impact direction of the particles in ecliptic coordinates (opposite to speed vector), both in the spacecraft-centered reference frame. The impact direction corresponds to the radiant of a meteor stream in a planetary atmosphere

conclude, the time intervals for the trail traverses given in Table 1 may be too conservative when taking into account particles smaller than $100 \mu \mathrm{m}$.

The IMEX model simulates relatively large particle sizes $r_{d} \geq 100 \mu \mathrm{m}$, much larger than the particles detectable by the CMDM dust detector $\left(r_{d} \geq 3 \mu \mathrm{m}\right)$. However, smaller particles are known to be present in the ejecta cloud of a comet, following a differential power law index of approximately -4 in their size distribution (Agarwal et al. 2010, and references therein), which corresponds to an index of approximately -3 for a cumulative size distribution. Their dynamical behaviour is not the same as for particles with $r_{d} \gtrsim 100 \mu \mathrm{m}$ due to the increasing effect of non-gravitational forces on the smaller particles. However, for sizes $10 \mu \mathrm{m} \leq \mathrm{r}_{\mathrm{d}} \leq 100 \mu \mathrm{m}$ their orbital characteristics are sufficiently similar to the larger particles, but spatially offset due to perturbations, as preliminary tests have demonstrated. An updated version of the IMEX Streams model for smaller particle sizes is planned, but currently not available for predictions.

To get to a more realistic representation of the expected fluxes, we consider two cases with a lower cutoff of $r_{\text {cutoff, } 1}=10 \mu \mathrm{m}$ and $r_{\text {cutoff }, 2}=30 \mu \mathrm{m}$, respectively. We extrapolate from the IMEX model flux of particles with sizes $r_{d} \geq 100 \mu \mathrm{m}$ using a cumulative power law size distribution index of -3 . This leads to an increase in total flux by a factor of 31.6 in the case of a cutoff at $30 \mu \mathrm{m}$, and a factor of 1000 for the $10 \mu \mathrm{m}$ cutoff. While it is unlikely that all particles in this size range remain in the stream, we consider a lower cutoff of 10 to $30 \mu \mathrm{m}$ conservative, and therefore a reasonable estimate for the expected fluxes and fluences overall. Based on the fluences listed in Table 1 (column 6), on the order of approximately 10 to 100 trail particle impacts are expected during traverses of the densest trails predicted by the IMEX model. This is also supported by our Helios observations mentioned above.

In addition to this, a comparison of the IMEX model to observations of cometary meteoroid streams by Soja et al. (2015b) concluded that the model likely underestimates the true fluxes by up to an order of magnitude, further increasing the odds for a successful detection of cometary streams.

Taking these considerations into account, our results indicate that, given its large sensitive area of $1 \mathrm{~m}^{2}$, CMDM has good chances to detect cometary meteoroid trail particles. Comets 21P/Giacobini-Zinner and 45P/H$\mathrm{M}-\mathrm{P}$ are the best candidates for trail detections during MMX' interplanetary transfer to Mars, while comets 114P/Wiseman-Skiff, 19P/Borrelly and 45P/H-M-P are particularly good candidates during the spacecraft's Martian orbital phase. Successful detections of individual 
cometary trails with CMDM and dust instruments on board other spacecraft will likely lead to an improved calibration of the IMEX Streams model and to more reliable flux predictions in the future.

The primary goal of the CMDM dust investigations at Mars will be the search for circum-Martian dust rings formed by particles originating from Phobos and Deimos (see, e.g. Zakharov et al. 2014; Spahn et al. 2019, for recent reviews). Given that MMX will be orbiting Mars in an orbit similar to Phobos during a significant fraction of the mission time, the spacecraft will largely be immersed in these rings and the question arises how CMDM can effectively separate cometary trail particles from ring particles. Dust number densities predicted for the Martian rings are approximately $6 \cdot 10^{-6} \mathrm{~m}^{-3}$ for the Phobos ring for particles larger than $30 \mu \mathrm{m}$ and approximately $10^{-4} \mathrm{~m}^{-3}$ for the Deimos ring for particles larger than $15 \mu \mathrm{m}$ (Krivov and Hamilton 1997; Krivov et al. 2006). Smaller particles are not expected to exist in abundance in the rings because their orbits are unstable. The expected particle impact speeds are in the range 0.2 to $0.8 \mathrm{~km} \mathrm{~s}^{-1}$ (Kobayashi et al. 2018a). Assuming a typical value of $0.5 \mathrm{~km} \mathrm{~s}^{-1}$, these number densities convert to dust fluxes of approximately $300 \mathrm{~m}^{-2}$ day $^{-1}$ and $4000 \mathrm{~m}^{-2}$ day $^{-1}$, respectively. Number densities for $100 \mu \mathrm{m}$ and bigger particles as simulated by IMEX are not given by Krivov and Hamilton (1997). However, from their results we estimate that the fluxes of such particles in the rings are likely more than one (in the case of Phobos) up to two (Deimos) orders of magnitude lower. Even these reduced numbers exceed the fluxes for cometary trail traverses predicted by our IMEX simulations by a few orders of magnitude. This makes particle identifications solely by enhanced dust fluxes very unlikely and, hence, we will have to use other criteria to identify cometary trail traverses. The impact speeds of trail particles exceed $10 \mathrm{~km} \mathrm{~s}^{-1}$ (Table 1) and are thus more than one order of magnitude higher than those of the ring particles. Furthermore, the impact direction of the particles can be derived from the spacecraft orientation at the particle impact time. Thus, the measured impact speed together with the impact direction will be the crucial parameters to separate cometary trail particles from Martian ring particles in the CMDM data set.

Similarly, during the interplanetary cruise phase of MMX, trail particles will have to be separated from the background flux of sporadic interplanetary particle impacts. Given that the impact speed will not serve as a crucial parameter because both populations will have similar speeds, we will instead search for cometary trail particles in the data set by using statistical techniques. We will use a method employed by Oberst and Nakamura (1991) to identify temporal clustering of meteoroid impacts detected by the Apollo lunar seismic network. The application of this method to in-situ dust detector data was described by Grün (2001) and successfully applied for the identification of Jovian dust stream particles in the Ulysses dust detector data set (Baguhl et al. 1993; Krüger et al. 2006).

About 15 years ago there was the claim that a meteor originating from comet 114P/Wiseman-Skiff was photographed in the Martian sky by the Mars exploration rover Spirit (Bell et al. 2004). However, later work quantified the effects of cosmic ray hits on the Spirit Pancam as part of a dedicated meteor search and placed this detection in doubt (Domokos et al. 2007). We also simulated the trail traverse of 114P/Wiseman-Skiff in 2004 with IMEX and we did not find a significantly enhanced dust flux in that period, consistent with the interpretation that the feature seen in the image taken by the Mars rover was a likely false detection.

In 2014 Mars experienced a very close encounter with comet C/2013 A1 (Siding Spring) at a distance of approximately $135,000 \mathrm{~km}$ (Withers 2014). On this occasion the planet traversed the cometary coma and meteoroid stream, leading to perturbations in Mars' atmosphere and ionosphere. The event created a temporary planet-wide ionospheric layer below Mars' main dayside ionosphere: The Neutral Gas and Ion Mass Spectrometer on board the Mars Atmosphere and Volatile EvolutioN spacecraft (MAVEN) detected a large variety of metal ions following the ablation of dust particles from the cometary coma ( $\mathrm{Na}, \mathrm{Mg}, \mathrm{Al}, \mathrm{K}, \mathrm{Ti}, \mathrm{Cr}, \mathrm{Mn}, \mathrm{Fe}, \mathrm{Co}, \mathrm{Ni}, \mathrm{Cu}, \mathrm{Zn}$, and possibly $\mathrm{Si}$ and $\mathrm{Ca}$; Benna et al. 2015). Intense ultraviolet emission due to magnesium and iron was attributed to dust trail particles approximately 1 to $100 \mu \mathrm{m}$ in size (Schneider et al. 2015). These observations showed that the ablation of meteoroids can lead to significant temporal changes in the composition of planetary atmospheres and ionospheres.

Comets with close encounters to Mars were studied by various authors (Adolfsson et al. 1996) (Treiman and Treiman 2000; Selsis et al. 2004; Christou 2010) (Christou and Vaubaillon 2011), see also Christou et al. (2019) for a recent review. These analyses studied the potential occurrences of meteor streams in the Martian atmosphere and derived impact speeds and radiant directions 
of the streams based on the encounter distance of the comet, but they were not able to make predictions for dust fluxes. In these works comets $24 \mathrm{P} /$ Schaumasse, 45P/H-M-P, 114P/Wiseman-Skiff and 275P/Hermann - among many other comets - were identified as potential candidates for sources of meteor streams in the Martian atmosphere. In our analysis, fluxes exceeding $10^{-3} \mathrm{~m}^{-2}$ day $^{-1}$ are predicted for these comets. Christou et al. (2019) list eight potential parent comets for Martian meteor showers in the time interval 2019 to 2021, with only $3 \mathrm{D} /$ Biela being in our list of predicted high flux candidates in the time interval 2024 to 2028 considered in our work. We also find many more comets with lower predicted fluxes in our simulations which we do not consider further as it is beyond the scope of this paper to make a detailed comparison of cometary trail traverses predicted by different methods. Finally, of the comets with high fluxes identified in our work, at least $45 \mathrm{P} / \mathrm{H}-$ M-P, 21P/Giacobini-Zinner and 3D/Biela are sources of known meteor showers in the Earth atmosphere (the $\alpha$ Capricornids, the Giacobinids and the Andromedids). Up to now the Earth remains the only planet where meteors have been observed (Christou et al. 2019).

Comet 3D/Biela is a lost comet as indicated by the letter "D". It most likely disintegrated in the early 1840 s, and two components were subsequently observed in 1846. The last observation of the two fragments occurred in 1852, and the comet has been lost since then (Kronk 2003). Comet Biela may have been the source of a very brilliant meteor shower in 1872, the Andromedids or Bielids. Nevertheless, meteoroids from this comet are likely still forming a meteoroid trail along the comet's former orbit as implied by the fact that its meteor shower is still active in the present time (e.g. Green 2013).

Comet 103P/Hartley (or Hartley 2) was the target of the Deep Impact extended (EPOXI) mission. Close-up images of this comet's nucleus revealed centimeter to decimeter sized objects in its vicinity (A'Hearn et al. 2011), at least a fraction of which is likely to become part of the comet's dust trail. Finally, in 2001 comet 19P/Borrelly was the target of the Deep Space 1 spacecraft.

Comet trails were the subject of sky surveys in the infrared (Sykes and Walker 1992; Reach et al. 2007; Arendt 2014) and recently also in the visible wavelength range (Ishiguro et al. 2007). Comet 103P/Hartley is the only comet identified in our analysis which is contained in any of these surveys but unfortunately its trail remained undetected (Reach et al. 2007).

We also performed IMEX simulations for the DES$\mathrm{TINY}^{+}$(Demonstration and Experiment of Space Technology for INterplanetary voYage with Phaethon flyby and dUst Science) mission to the asteroid (3200) Phaethon (Kawakatsu and Itawa 213; Arai et al. 2018) scheduled for launch in 2024. Our simulations show that along its presently planned interplanetary trajectory the spacecraft will cross the trail of comet $45 \mathrm{P} / \mathrm{H}-\mathrm{M}-\mathrm{P}$, giving the Dust Analyzer on board (Kobayashi et al. 2018b) the possibility to detect and analyze individual particles originating from this comet.

For our IMEX simulations we used a candidate trajectory for the interplanetary transfer of MMX to Mars which is the current nominal spacecraft trajectory (Campagnola et al. 2018). Any change in the MMX launch date will affect the actual trajectory, and thus likely lead to a different set of cometary trail traverses. On the other hand, for the Mars orbital phase we used Mars as the target and these simulation results are valid independent of the spacecraft under consideration. They may give useful information about the occurrence of meteors also for other missions carrying landers or rovers to the red planet. For MMX they remain useful as long as the spacecraft's actual operational phase at Mars is at least partially covered by our simulations.

The fact that we performed our simulations for the MMX Martian orbital phase with Mars as a target may lead to deviations in the impact directions and speeds as compared to those given in Table 1 because the spacecraft will be in an orbit very similar to the one of Phobos. Considering Phobos' orbital speed of about $2 \mathrm{~km} \mathrm{~s}^{-1}$, the uncertainty in the impact speed caused by this approximation should be below 20\%. Furthermore, considering that CMDM is a flat sensor with $2 \pi$ field-of-view, the error in the impact direction should not lead to a significant uncertainty in the dust flux, in particular when taking into account that the fluxes predicted by IMEX have an uncertainty of at least a factor of ten anyway. For a more detailed analysis of the impact directions and speeds, and in particular for planning the CMDM measurements at Mars, it will be worthwhile to use the actual MMX trajectory around Mars in the future.

Cometary meteoroid impacts may pose significant risks for Earth orbiting satellites and crewed spaceflight as has been already mentioned in the Introduction. For crewed missions to other planets this will likely become 
an increasingly severe issue because the spacecraft will become increasingly larger and long flight times will be required which will increase the probability for hazardous meteoroid hits. Christou and Vaubaillon (2010) pointed out that in the future the impact hazard should already be considered during the analysis and design phase of interplanetary missions in order to optimize their trajectories and reduce hazardous cometary trail traverses as much as possible.

\section{Conclusions}

We used the IMEX dust streams in space model ( Soja et al. 2015b) to predict cometary meteoroid trail traverses by the MMX spacecraft. The model simulates cometary trails in the inner Solar System formed by particles in the size range $10^{-8} \mathrm{~kg}$ to $10^{-2} \mathrm{~kg}$, approximately corresponding to $100 \mu \mathrm{m}$ to $1 \mathrm{~cm}$ particle radius.

Using the presently available nominal trajectory of MMX we found that during its interplanetary transfer to Mars and while being in Mars orbit the spacecraft will traverse several cometary meteoroid trails. The highest dust flux is predicted for October 2026 when MMX will be in Mars orbit traversing the trail of comet 114P/ Wiseman-Skiff, with a maximum flux of $0.15 \mathrm{~m}^{-2}$ day $^{-1}$ lasting for about three days. We find a total of 12 trail traverses during the entire mission, with fluxes of at least $10^{-3} \mathrm{~m}^{-2}$ day $^{-1}$, covering a total time period of approximately 90 days. Smaller particles-which are not covered by the present IMEX model-are expected to exist in the comet trails as well and may lead to a few orders of magnitude higher dust fluxes. We conclude that the large area CMDM dust sensor on board MMX with its detection threshold of approximately $3 \mu \mathrm{m}$ particle radius will likely be able to detect trail particles during a few meteoroid trail traverses.

The in situ detection and analysis of cometary trail particles in space creates a new opportunity to remotely measure the composition of celestial bodies without the necessity to fly a spacecraft to or even land on the source objects. Missions carrying dust instruments in the near future that may benefit from this approach include DESTINY ${ }^{+}$to the asteroid (3200) Phaethon, Europa Clipper to the Jupiter system and the Interstellar Mapping and Acceleration Probe (Kobayashi et al. 2018b; Kempf 2018; McComas et al. 2018). Successful measurements of meteoroid trails by spaceborne instruments may lead to improvements of cometary trail models. Forecasts of trail traverses by planets in connection with in situ or remote spacecraft investigations can also provide new insights into atmospheric and ionospheric processes. Last but not least, they can make essential

Table 2 Orbital elements of comets discussed in this paper from the JPL Small Body Database (ssd.jpl.nasa.gov)

\begin{tabular}{|c|c|c|c|c|c|c|c|c|}
\hline Comet & e & $\begin{array}{l}9 \\
{[\mathrm{AU}]}\end{array}$ & $\begin{array}{l}i \\
{\left[{ }^{\circ}\right]}\end{array}$ & $\begin{array}{l}\Omega \\
{\left[{ }^{\circ}\right]}\end{array}$ & $\begin{array}{l}\omega \\
{\left[{ }^{\circ}\right]}\end{array}$ & tPerihelion & $\begin{array}{l}\text { Orbital Period } \\
{[y r]}\end{array}$ & Epoch \\
\hline (1) & (2) & (3) & (4) & (5) & (6) & (7) & (8) & (9) \\
\hline 3D/Biela & 0.75 & 0.88 & 13.2 & 250.7 & 221.7 & 26.11 .1832 & 6.65 & 03.12 .1832 \\
\hline 19P/Borrelly & 0.62 & 1.36 & 30.3 & 75.4 & 353.4 & 14.09 .2001 & 6.86 & 01.05 .2004 \\
\hline 21P/Giacobini-Zinner & 0.71 & 1.01 & 32.0 & 195.4 & 172.8 & 10.09 .2018 & 6.55 & 05.11 .2017 \\
\hline 24P/Schaumasse & 0.70 & 1.21 & 11.7 & 79.7 & 58.0 & 16.11 .2017 & 8.26 & 24.11 .2015 \\
\hline 45P/H-M-P & 0.82 & 0.53 & 4.2 & 89.0 & 326.3 & 31.12 .2016 & 5.26 & 14.02 .2017 \\
\hline 103P/Hartley & 0.69 & 1.06 & 13.6 & 219.8 & 181.2 & 28.10.2010 & 6.46 & 30.11 .2010 \\
\hline 114P/Wiseman-Skiff & 0.55 & 1.58 & 18.3 & 271.1 & 172.8 & 14.01 .2020 & 6.67 & 13.03 .2018 \\
\hline 185P/Petriew & 0.70 & 0.93 & 14.0 & 214.1 & 181.9 & 13.08.2012 & 5.46 & 30.06 .2013 \\
\hline 255P/Levy & 0.67 & 1.00 & 18.3 & 279.7 & 179.7 & 02.05 .2017 & 5.30 & 20.11 .2014 \\
\hline 275P/Hermann & 0.71 & 1.65 & 21.6 & 349.3 & 173.1 & 19.02.1999 & 13.84 & 06.03 .2004 \\
\hline
\end{tabular}




\section{predictions for spacecraft hazards for future crewed deep space missions.}

\section{Appendix \\ See Table 2.}

\section{Acknowledgements}

The IMEM model and the IMEX Dust Streams in Space model were developed under ESA funding (contracts 21928/08/NL/AT and 4000106316/12/NL/AF IMEX). We are grateful to the MPI für Sonnensystemforschung, Chiba Institute of Technology and the University of Stuttgart for their support. H. Kimura gratefully acknowledges support by the Grants-in-Aid for Scientific Research (KAKENHI number 19H05085) of the Japan Society for the Promotion of Science (JSPS). We are grateful to two anonymous referees whose comments substantially improved the presentation of our results. We are grateful to Rachel Soja, formerly at the University of Stuttgart, who was one of the main developers of the IMEX model. We are also grateful to Valeri Dikarev, main developer of the IMEM model, for support on the interpretation of the IMEM model results. For authors'funding see below.

\section{Authors' contributions}

HK: Performed most of the IMEX simulations, writing of the manuscript, discussion and interpretation of results. MK: Provided technical information of the CMDM instrument and MMX mission necessary for the interpretation of results in view of actual detection conditions with CMDM. PS: Development and maintenance of the IMEX model, discussion and interpretation of results w.r.t. particle dynamics and dust fluxes. GM-K: Preparation of spacecraft trajectory data, interpretation of results. MS: Development and maintenance of the IMEX model, discussion of the simulation results w.r.t. particle dynamics and dust fluxes. Critical checks of IMEM model. HK: Discussion and interpretation of the results on particle dynamics. EG: Supervision of the development of the IMEX model. Discussion and interpretation of the results with emphasis on past and future dust trail measurements. RS: Supervision of the development of the IMEM and IMEX modela. Discussion and interpretation of the results with emphasis of future spaceborne dust trail measurements. All authors made significant contributions during the preparation of the manuscript in interpreting and discussing the simulation results, and finalising the manuscript. All authors read and approved the final manuscript.

\section{Funding}

Open Access funding enabled and organized by Projekt DEAL. The IMEM model and the IMEX Dust Streams in Space model were developed under ESA funding (contracts 21928/08/NL/AT and 4000106316/12/NL/AF - IMEX). H. Krüger was supported by Max-Planck-Institut für Sonnensystemforschung and by Chiba Institute of Technology during a research visit during which part of the computer simulation work and manuscript writing was performed. $\mathrm{H}$. Kimura gratefully acknowledges support by the Grants-in-Aid for Scientific Research (KAKENHI number 19H05085) of the Japan Society for the Promotion of Science (JSPS). R. Srama, M. Sommer, G. Moragas-Klostermeyer and P. Strub acknowledge support by ESA, the University of Stuttgart and by a large number of volunteers who provided their computers for extensive simulation work under shared computing.

\section{Availability of data and materials}

Not applicable.

\section{Declarations}

\section{Competing interests}

There are no competing interests.

\section{Author details}

${ }^{1}$ MPI für Sonnensystemforschung, Göttingen, Germany. ${ }^{2}$ PERC, Chiba Institute of Technology, Narashino, Japan. ${ }^{3}$ Institut für Raumfahrtsysteme, Universität Stuttgart, Stuttgart, Germany. ${ }^{4}$ Max-Planck-Institut für Kernphysik, Heidelberg, Germany. ${ }^{5}$ LASP, University of Colorado, Boulder, CO, USA. ${ }^{6}$ Baylor University, Waco, TX, USA.
Received: 27 November 2020 Accepted: 29 March 2021

Published online: 16 April 2021

\section{References}

Adolfsson LG, Gustafson BÅS, Murray CD (1996) The Martian atmosphere as a meteoroid detector. Icarus 119(1):144-152

Agarwal J, Müller M, Grün E (2007) Dust environment modelling of Comet 67P/Churyumov-Gerasimenko. Space Sci Rev 128:79-131

Agarwal J, Müller M, Reach WT, Sykes MV, Böhnhardt H, Grün E (2010) The dust trail of Comet 67P/Churyumov-Gerasimenko between 2004 and 2006. Icarus 207:992-1012

A'Hearn MF, Belton MJS, Delamere WA, Feaga LM, Hampton D, Kissel J, Klaasen KP, McFadden LA, Meech KJ, Melosh HJ, Schultz PH, Sunshine JM, Thomas PC, Veverka J, Wellnitz DD, Yeomans DK, Besse S, Bodewits D, Bowling TJ, Carcich BT, Collins SM, Farnham TL, Groussin O, Hermalyn B, Kelley MS, Kelley MS, Li J-Y, Lindler DJ, Lisse CM, McLaughlin SA, Merlin F, Protopapa S, Richardson JE, Williams JL (2011) EPOXI at Comet Hartley 2. Science 332:1396-1400

A'Hearn MF, Millis RC, Schleicher DO, Osip DJ, Birch PV (1995) The ensemble properties of comets: results from narrowband photometry of 85 comets, 1976-1992. Icarus 118(2):223-270

Altobelli N, Grün E, Landgraf M (2006) A new look into the Helios dust experiment data: presence of interstellar dust inside the Earth's orbit. Astron Astrophys 448:243-252

Arai T, Kobayashi M, Ishibashi K, Yoshida F, Kimura H, Wada K, Senshu H, Yamada M, Okudaira O, Okamoto T, Kameda S, Srama R, Krüger H, Ishiguro M, Yabuta H, Nakamura T, Watanabe J, Ito T, Ohtsuka K, Tachibana S, Mikouchi T, Komatsu M, Nakamura-Messenger K, Sasaki S, Hiroi T, Abe S, Urakawa S, Hirata N, Demura H, Komatsu G, Noguchi T, Sekiguchi T, Inamori T, Yano H, Yoshikawa M, Ohtsubo T, Okada T, Iwata T, Nishiyama K, Toyota H, Kawakatsu, Y, Takashima T (2018) Destiny+ Mission: Flyby of Geminids Parent Asteroid (3200) Phaethon and in-situ Analyses of Dust Accreting on the Earth. In Lunar and Planetary Institute Science Conference Abstracts, volume 49 of Lunar and Planetary Institute Science Conference Abstracts, page 2570

Arendt RG (2014) DIRBE Comet Trails. Astronomical J 148(6):135

Baguhl M, Grün E, Linkert G, Linkert D, Siddique N (1993) Identification of 'small' dust impacts in the Ulysses dust detector data. Planet Space Sci 41:1085-1098

Beech M, Brown P, Jones J (1995) The potential danger to space platforms from meteor storm activity. Quart J R Astron Soc 36:127

Bell JF, Squyres SW, Arvidson RE, Arneson HM, Bass D, Blaney D, Cabrol N, Calvin W, Farmer J, Farrand WH, Goetz W, Golombek M, Grant JA, Greeley R, Guinness E, Hayes AG, Hubbard MYH, Herkenhoff KE, Johnson MJ, Johnson JR, Joseph J, Kinch KM, Lemmon MT, Li R, Madsen MB, Maki JN, Malin M, McCartney E, McLennan S, McSween HY, Ming DW, Moersch JE, Morris RV, Noe Dobrea EZ, Parker TJ, Proton J, Rice JW, Seelos F, Soderblom J, Soderblom LA, Sohl-Dickstein JN, Sullivan RJ, Wolff MJ, Wang A (2004) Pancam multispectral imaging results from the Spirit Rover at Gusev Crater. Science 305(5685):800-807

Benna M, Mahaffy PR, Grebowsky JM, Plane JMC, Yelle RV, Jakosky BM (2015) Metallic ions in the upper atmosphere of Mars from the passage of comet C/2013 A1 (Siding Spring). Geophys Res Lett 42(12):4670-4675

Cameron RA, Brissenden RJ, Bucher S, Gage K, Martin E, Shropshire D, Virani SN (2004) A possible meteoroid impact on the Chandra X-Ray Observatory during the 2003 Leonids. In 35th COSPAR Scientific Assembly, volume 35, page 4490

Campagnola S, Yam CH, Tsuda Y, Ogawa N, Kawakatsu Y (2018) Mission analysis for the Martian Moons Explorer (MMX) mission. Acta Astronautica 146:409-417

Caswell RD, McBride N, Taylor AD (1995) Olympus end of life anomaly-A Perseid meteoroid event? Int J Impact Eng 17:139-150

Christou A, Vaubaillon J, Withers P, Hueso R, Killen R (2019) Extra-Terrestrial Meteors, pages 119-135. ed. Ryabova, Galina O. and Asher, David J. and Campbell-Brown, Margaret J., Cambridge, Cambridge University Press

Christou AA (2010) Annual meteor showers at Venus and Mars: lessons from the Earth. Monthly Notices R Astro Soc 402(4):2759-2770 
Christou AA, Vaubaillon J (2010) Modelling an encounter between a spacecraft and a cometary meteoroid trail in interplanetary space: the case of the Venus Climate Orbiter and comet 27P/Crommelin. Planet Space Sci 58(7-8):1026-1034

Christou AA, Vaubaillon J (2011) Numerical Modeling of Cometary Meteoroid Streams Encountering Mars and Venus. In Cooke, W. J., Moser, D. E., Hardin, B. F., and Janches, D., editors, Meteoroids: The Smallest Solar System Bodies, page 26

Crifo JF, Rodionov AV (1997) The dependence of the circumnuclear coma structure on the properties of the nucleus. Icarus 127:319-353

Dikarev V, Grün E, Baggaley J, Galligan D, Landgraf M, Jehn R (2005) The new ESA meteoroid model. Adv Space Res 35:1282-1289

Divine N and Newburn RL (1987) Modeling Halley before and after the encounters. Astron Astrophys 187(1-2):867-872

Domokos A, Bell JF, Brown P, Lemmon MT, Suggs R, Vaubaillon J, Cooke W (2007) Measurement of the meteoroid flux at Mars. Icarus 191(1):141-150

Green DWE (2013) Andromedid Meteors. Central Bureau Electr Telegr 3741:1

Grün E (2001) In situ measurements of cosmic dust. In: Grün E, Gustafson BAS, Dermott SF, Fechtig H (eds) Interplanetary Dust. Springer Verlag, Berlin Heidelberg New York, pp 295-346

Grün E, Krüger H, Srama R (2019) The dawn of dust astronomy. Space Sci Rev 215(7):46

Grün E, Pailer N, Fechtig H, Kissel J (1980) Orbital and physical characteristics of micrometeoroids in the inner solar system as observed by helios 1. Planet Space Sci 28:333-349

Güttler C, Mannel T, Rotundi A, Merouane S, Fulle M, Bockelée-Morvan D, Lasue J, Levasseur-Regourd AC, Blum J, Naletto G, Sierks H, Hilchenbach M, Tubiana C, Capaccioni F, Paquette JA, Flandes A, Moreno F, Agarwal J, Bodewits D, Bertini I, Tozzi GP, Hornung K, Langevin Y, Krüger H, Longobardo A, Della Corte V, Tóth I, Filacchione G, Ivanovski SL, Mottola S, Rinaldi G (2019) Synthesis of the morphological description of cometary dust at comet 67P/Churyumov-Gerasimenko. Astron Astrophys 630:A24

Hamilton DP (1996) The asymmetric time-variable rings of mars. Icarus 119:153-172

Ishiguro M, Sarugaku Y, Ueno M, Miura N, Usui F, Chun M-Y, Kwon SM (2007) Dark red debris from three short-period comets: 2P/Encke, 22P/Kopff, and 65P/Gunn. Icarus 189(1):169-183

Ishimoto H, Kimura H, Nakagawa N, Mukai T (1997) Planned observation of Phobos/Deimos dust rings by PLANET-B. Adv Space Res 19(1):123-126

Jorda L, Crovisier J, Green DWE (2008) The correlation between visual magnitudes and water production rates. In Asteroids Comets Meteors $2008,1405,8046$

Kawakatsu Y, Itawa T (2013) Destiny mission overview: a small satellite mission for deep space exploration technology demonstration. Adv Astron Sci 146:12-585

Kempf S (2018) The Surface Dust Analyzer (SUDA) on Europa Clipper. In European Planetary Science Congress, pages EPSC2018-462

Kimura H, Hilchenbach M, Merouane S, Paquette J, Stenzel O (2020) The morphological, elastic, and electric properties of dust aggregates in comets: a close look at COSIMA/Rosetta's data on dust in comet 67P/ Churyumov-Gerasimenko. Planet Space Sci 181:104825

Kimura H, Wada K, Yoshida F, Hong P, Senshu H, Arai T, Hirai T, Kobayashi M, Ishibashi K, Yamada M (2020b) The tensile strength of dust aggregates consisting of small elastic grains: Constraints on the size of condensates in protoplanetary disks. Monthly Notices of the Royal Astro Soc 496:1667

Kobayashi M, Krüger H, Senshu H, Wada K, Okudaira O, Sasaki S, Kimura H (2018a) In situ observation of dust particles of Martian dust belts by a large-sensitive-area dust sensor. Planet Space Sci 156:41-46

Kobayashi M, Okudaira O, Kurosawa K, Okamoto T, Matsui T (2016) Dust Sensor with Large Detection Area Using Polyimide Film and Piezoelectric Elements. In 3rd International Workshop on Instrumentation for Planetary Mission, volume 1980, page 4047

Kobayashi M, Srama R, Krüger H, Arai T, Kimura H (2018b) Destiny+ Dust Analyser. In Lunar and Planetary Institute Science Conference Abstracts, volume 49 of Lunar and Planetary Institute Science Conference Abstracts, page 2050

Koschny D, Soja RH, Engrand C, Flynn GJ, Lasue J, Levasseur-Regourd A-C, Malaspina D, Nakamura T, Poppe AR, Sterken VJ, Trigo-Rodríguez JM (2019) Interplanetary dust, meteoroids, meteors and meteorites. Space Sci Rev 215(4):34
Krivov AV, Feofilov AG, Dikarev VV (2006) Search for the putative dust belts of Mars: the late 2007 opportunity. Planet Space Sci 54:871-878 Krivov AV, Hamilton DP (1997) Martian dust belts: Waiting for discovery, Icarus 128:335-353

Kronk GW (2003) Cometography: a Catalog of Comets, vol 2. Cambridge University Press, Cambridge, pp 1800-1899

Krüger H, Graps AL, Hamilton DP, Flandes A, Forsyth RJ, Horányi M, Grün E (2006) Ulysses jovian latitude scan of high-velocity dust streams originating from the jovian system. Planet Space Sci 54:919-931

Krüger H, Strub P, Sommer M, Altobelli N, Kimura H, Lohse A-K, Grün E, Srama R (2020) Helios spacecraft data revisited: Detection of cometary meteoroid trails by following in-situ dust impacts. Astronomy and Astrophysics (in press)

Kuramoto K, Kawakatsu Y, Fujimoto M (2019) Martian Moons eXploration (MMX): architecture of its science. In EPSC-DPS Joint Meeting 2019, volume 2019, pages EPSC-DPS2019-1636

Kuramoto K, Kawakatsu Y, Fujimoto M, Genda H, Imamura T, Kameda S, Matsumoto K, Miyamoto H, Morota T, Nagaoka H, Nakamura T, Ogawa K, Otake H, Ozaki M, Sasaki S, Senshu H, Tachibana S, Terada N, Usui T, Wada K, Watanabe S, MMX Study Team (2018) Martian Moons Exploration (MMX) Conceptual Study Update. In Lunar and Planetary Science Conference, volume 49 of Lunar and Planetary Science Conference, page 2143

Levasseur-Regourd A-C, Agarwal J, Cottin H, Engrand C, Flynn G, Fulle M, Gombosi T, Langevin Y, Lasue J, Mannel T, Merouane S, Poch O, Thomas N, Westphal A (2018) Cometary dust. Space Sci Rev 214(3):64

McComas DJ, Christian ER, Schwadron NA, Fox N, Westlake J, Allegrini F, Baker DN, Biesecker D, Bzowski M, Clark G, Cohen CMS, Cohen I, Dayeh MA, Decker R, de Nolfo GA, Desai MI, Ebert RW, Elliott HA, Fahr H, Frisch PC, Funsten HO, Fuselier SA, Galli A, Galvin AB, Giacalone J, Gkioulidou M, Guo F, Horanyi M, Isenberg P, Janzen P, Kistler LM, Korreck K, Kubiak MA, Kucharek H, Larsen BA, Leske RA, Lugaz N, Luhmann J, Matthaeus W, Mitchell D, Moebius E, Ogasawara K, Reisenfeld DB, Richardson JD, Russell CT, Sokół JM, Spence HE, Skoug R, Sternovsky Z, Swaczyna P, Szalay JR, Tokumaru M, Wiedenbeck ME, Wurz P, Zank GP, Zirnstein EJ (2018) Interstellar Mapping and Acceleration Probe (IMAP): a new NASA mission. Space Sci Rev 214(8):116

Oberst J, Nakamura Y (1991) A search for clustering among the meteoroid impacts detected by the Apollo Lunar Seismic Network. Icarus 91:315-325

Ogawa N, Tsuda Y, Takei Y, Inoue H, Takahashi S, Kawakatsu Y (2019) Orbit design for the Martian moons exploration mission. Trans JSASS Aerospace Tech Japan 17:398-403

Reach WT, Kelley MS, Sykes MV (2007) A survey of debris trails from shortperiod comets. Icarus 191(1):298-322

Schneider NM, Deighan JI, Stewart AIF, MCClintock WE, Jain SK, Chaffin MS, Stiepen A, Crismani M, Plane JMC, Carrillo-Sánchez JD, Evans JS, Stevens MH, Yelle RV, Clarke JT, Holsclaw GM, Montmessin F, Jakosky BM (2015) MAVEN IUVS observations of the aftermath of the Comet Siding Spring meteor shower on Mars. Geophys Res Lett 42(12):4755-4761

Selsis F, Brillet J, Rapaport M (2004) Meteor showers of cometary origin in the Solar System: revised predictions. Astron Astrophys 416:783-789

Showalter MR, Hamilton DP, Nicholson PD (2006) A deep search for Martian dust rings and inner moons using the Hubble Space Telescope. Planet Space Sci 54:844-854

Soja RH, Grün E, Strub P, Albin T, Millinger M, Vaubaillon J, Alius W, Camodeca G, Hein F, Laskar J, Gastineau M, Fienga A, Sommer M, Schwarzkopf GJ, Herzog J, Gutsche K, Skuppin N, Srama R (2019) IMEM2: a meteoroid environment model for the inner solar system. Astron Astrophys 628:A109

Soja RH, Herzog JT, Sommer M, Rodmann J, Vaubaillon J, Strub P, Albin T, Sterken V, Hornig A, Bausch L, Grün E, Srama R (2015a) Meteor storms and showers with the IMEX model. In: Rault J-L, Roggemans P (eds) International Meteor Conference Mistelbach. Austria, pp 66-69

Soja RH, Sommer M, Herzog J, Agarwal J, Rodmann J, Srama R, Vaubaillon J, Strub P, Hornig A, Bausch L, Grün E (2015b) Characteristics of the dust trail of 67P/Churyumov-Gerasimenko: an application of the IMEX model. Astron Astrophys 583:A18

Soter S (1971) The dust belts of Mars. Technical report, Center for Radiophysics and Space Research Report No, p 462 
Spahn F, Sachse M, Seiß M, Hsu H-W, Kempf S, Horányi M (2019) Circumplanetary dust populations. Space Sci Rev 215(1):11

Strub P, Sterken VJ, Soja R, Krüger H, Srama R (2019) Heliospheric modulation of the interstellar dust flow on to Earth. Astron Astrophys 621:A54

Sykes MV, Lebofsky LA, Hunten DM, Low F (1986) The discovery of dust trails in the orbits of periodic comets. Science 232(4754):1115-1117

Sykes MV, Walker RG (1992) Cometary dust trails I. Survey. Icarus 95(2):180-210

Topputo F, Belbruno E (2015) Earth-Mars transfers with ballistic capture. Celestial Mech Dyn Astron 121(4):329-346

Treiman AH, Treiman JS (2000) Cometary dust streams at Mars: preliminary predictions from meteor streams at Earth and from periodic comets. $J$ Geophys Res 105(E10):24571-24582
Withers P (2014) Predictions of the effects of Mars's encounter with comet C/2013 A1 (Siding Spring) upon metal species in its ionosphere. Geophys Res Lett 41(19):6635-6643

Zakharov A, Horanyi M, Lee P, Witasse O, Cipriani F (2014) Dust at the Martian moons and in the circummartian space. Planet Space Sci 102:171-175

\section{Publisher's Note}

Springer Nature remains neutral with regard to jurisdictional claims in published maps and institutional affiliations.

\section{Submit your manuscript to a SpringerOpen ${ }^{\circ}$ journal and benefit from:}

- Convenient online submission

- Rigorous peer review

- Open access: articles freely available online

- High visibility within the field

- Retaining the copyright to your article

Submit your next manuscript at $\boldsymbol{\nabla}$ springeropen.com 